\title{
THE EQUILIBRIA OF STEREOISOMERS, II
}

\section{BY WILIER D. BANCROFT}

In the first paper ${ }^{1}$ of the series, it was shown how Duliem's theory of permanent changes offered a satisfactory explanation of many phenomena connected with the change of one isomer into another. In this paper I shall discuss some of the changes due to the addition of one or more components. The cases to be treated may be classified as follows:

I. The new component forms no compound either with the $\alpha$ or the $\beta$ modification.

I. No reversal of stability.

a. The $\beta$ modification changes into the $a$ modification without heat effect.

b. The $\beta$ modification changes into the $\alpha$ modification with evolntion of heat.

c. The $\beta$ modification changes into the a modification with absorption of heat.

2. Reversal of stability.

a. Without displacement of equilibrium.

b. With displacement of equilibrium.

It is obrious that a system coming under any of these heads will behave like a three-component system if we work rapidly: and like a two-component system if we wait for the final equilibrium to be reached. We will begin with the case where the new component $\mathrm{C}$ forms no compound either with the $\alpha$ or the $\beta$ modification, making the further proviso that the $\alpha$ modification

'Jour. Phys. Chem. 2, I43 (IS98). 
shall be the stable form under all circumstances. The general diagrams for cases $I a, I b$ and $I c$ are given in Fig. I.

Working rapidly, we shall be able to realize the three boundary curves: DO with the $\alpha$ and the $\beta$ modifications as solid phases; $\mathrm{HO}$ with the $\alpha$ modification and the component $\mathrm{C}$ as solid phases; $\mathrm{EO}$ with the $\beta$ modification and the component $\mathrm{C}$ as solid phases. The a modification will be solid phase in the field $\mathrm{ADOH}$; the $\beta$ modification in the field $\mathrm{BDOE}$; the component $\mathrm{C}$ in the field $\mathrm{CEOH}$. At the point $\mathrm{O}$ we shall have the two

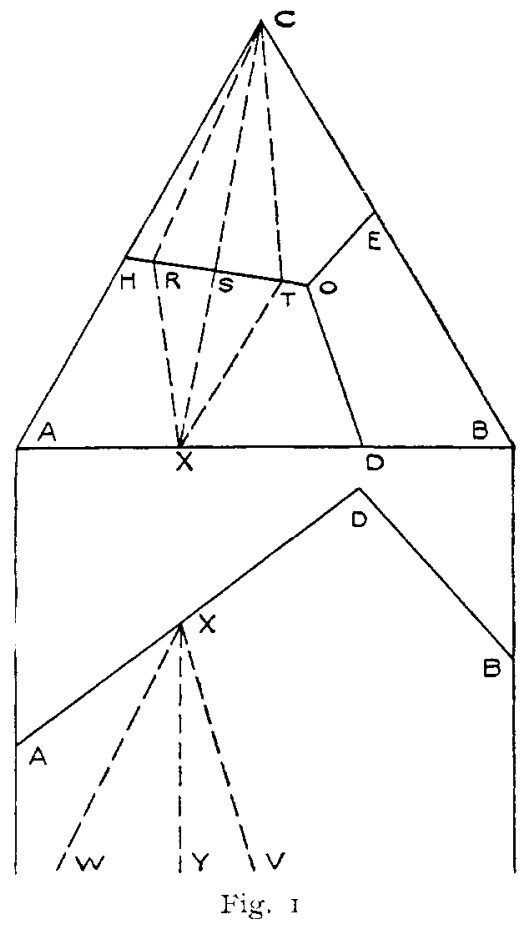

modifications and the component $\mathrm{C}$ temporarily in equilibrinm with solution and vapor. If, however, we wait until equilibrium is reached, the variance of the system drops from three to two; fields become lines; lines become points and points disappear. The point $O$ ceases to be a possibility; the line ADB becomes the point $\mathrm{X}$, while the surface of the triangle dwindles to a line 
connecting $\mathrm{X}$ and $\mathrm{C}$. It is this line which interests us. If the equilibrim between the $\alpha$ and $\beta$ modifications is independent of the temperature, as shown by the line $\mathrm{YX}$, and if this equilibrium be affected but little or not at all by the component $\mathrm{C}$, the boundary curves will form an approximately straight line from $\mathrm{X}$ to $\mathrm{C}$, in other words XSC. If the component $\mathrm{C}$ displace the equilibrium to the side of the $\beta$ modification the point $\mathrm{S}$ will move to the right along $\mathrm{HO}$ while it will move to the left along the same line in case the equilibrinm be displaced to the side of the a modification.

The line XSC does not cut the field BDOE. Consequently; the $\beta$ modification will not be stable under any circumstances, and the diagram conforms to the liniting condition imposed. Along the line XS the solid phase will be the a modification; along $C S$ the component $C$ will be solid phase; the point $S$ will represent the stable quadruple point at which the a modification and the component $\mathrm{C}$ are in stable equilibrium, as solid phases, with solution and rapor. The temperature will rise in both directions from the stable quadruple point $S$ along the boundary curves to $\mathrm{X}$ and $\mathrm{C}$. In considering the effect of keeping a definite initial solution at a definite temperature, we must bear in mind that the temperature of the point $S$ is necessarily higher than that of the point $\mathrm{O}$ and lower than that of $\mathrm{H}$; but that it may be either ligher or lower than that of $\mathrm{D}$ and of $\mathrm{E}$. If, as is often the case, the temperature of the point $\mathrm{X}$ is higher than the melting-point of $\mathrm{C}$, the temperature of $\mathrm{S}$ will usually be higher than that of $\mathrm{E}$ and lower than that of $\mathrm{O}$.

If there is no solid phase, the change of concentration at constant temperature will be such that the system passes along a line parallel to the base of the triangle in the direction of the 'natural' surface. The lines IXSC all lie in this surface which is the locus of all states of stable equilibrium that include solution and rapor. Starting with a solntion represented by sone point on BEC the final result will be unsaturated solution and vapor if the temperature of $\mathrm{E}$ is higher than that of $\mathrm{S}$. If the temperature of $\mathrm{E}$ be lower than that of $\mathrm{S}$ there will be some temperature for which an isothermal line parallel to the base of 
the triangle will cut both $\mathrm{BE}$ and XS. At this temperature, a solution saturated with respect to the $\beta$ modification will change to a solution saturated with respect to the a modification. At constant temperature all solutions represented by points on $\mathrm{BEC}$ which contain more of the $\beta$ modification than this one will form solutions unsaturated with respect to the a modification while all solutions represented by points on $\mathrm{BEC}$, which contain less of the $\beta$ modification than this one will change to solutions supersaturated with respect to the a modification, to the component $\mathrm{C}$ or to both these plases. In the first case the resulting system will be the solid a modification, solution and rapor, the composition of the solution being represented by the point on XS for the temperature in question. In the second case the final system will be solid $\mathrm{C}$, solution and rapor, the composition of the solution being given by the point on CS corresponding to the temperature of the experiment. In case the resulting solntion is stupersaturated with respect to both solid phases, the phases finally in equilibrium will be the two solids and rapor.

Starting with a saturated solution represented by a point on $\mathrm{AHC}$, the final equilibrium reached, at constant temperature, will always be unsaturated solution and rapor.

If the initial system be solid, solntion and rapor instead of saturated solution and rapor, the change in the composition of the solution will no longer be represented by a line parallel to the base of the triangle. As long as the solid phase is present the system will pass along the isotherm for solid, solution and vapor; if the solid phase disappear at any point, the change from then on will be represented, as before, by a line parallel to the base of the triangle. Starting, with an excess of the $\beta$ modification, from a point on $B E$ haring a temperature higher than that of the point $D$, there will be gradual decrease of the solid phase, the composition of the solution approximating more and more closely to that of the point on BD for the same temperature. Since the amount of the component $C$ in the solution can never become zero, the solid $\beta$ modification will disappear eventually and we shall have saturated solution and rapor, the changes of which have just been discussed at length. If we start, 
with an excess of the $\beta$ modification from a point on $\mathrm{BE}$, having a temperature lower than that of $\mathrm{D}$, the system will pass along the isotherm, the concentration changing until, at the point where the isotherm cuts OD, the a modification appears as solid phase. The concentration will then remain constant until the whole of the $\beta$ modification has changed into the a modification. The concentration will then vary until the solution has the composition of the point at which the isotherm cuts XS, the final system being the solid a modification, solution and vapor.

If we start, with an excess of the component $C$, from a point on $\mathrm{CE}$ or $\mathrm{OH}$ the final equilibrinn will be, ontwardly, the same as the initial one; namely, solid $C$, solution and vapor. The difference will be in the composition of the solution and, possibly; in the total amonnt of the solid phase. It is worth noting that if the lowering of the freezing-point of $\mathrm{C}$ due to a mixture of the $\alpha$ and the $\beta$ modifications were the sum of the partial depressions of the modifications taken singly; the part of the isotherm in the field for C would be a straight line parallel to the base of the triangle. If this were true, the surface lying between $\mathrm{HC}$ and $\mathrm{EC}$ would be absolutely plane. It seems more probable, however, that the line CS lies below the plane passing through the lines HCE, in which case the isotherm will slant up towards $\mathrm{C}$ and the total anount of the solid $\mathrm{C}$ will decrease as the systems under consiceration pass from their initial to their final states. Expressed in other words, the sum of the partial depressions of the freezing-point is less than the total depression due to the mixture.

If we start from the quadruple point $\mathrm{E}$, with an excess of both solid phases, there will be, at first, no change in concentration. If the component $\mathrm{C}$ be present in excess, the $\beta$ modification will disappear first and the system will belave in the same general way as any system represented by some point between $C$ and $E$. If the modification be present in excess, the component $\mathrm{C}$ will be the first to disappear and the systenn will behave in the same general way as any system represented by some point between $\mathrm{B}$ and $\mathrm{E}$.

If we start, with an excess of the a modification, from any 
point on $\mathrm{AH}$, the final state will differ from the initial one in that some of the solid phase will have passed into solution. Starting from any point inside the triangle at a temperature below that of the point $S$ and keeping the temperature constant, the phases, at equilibrium, will be the a modification, the connponent $\mathrm{C}$ and rapor.

The effect of changing the temperature is rery simple. While the temperature rises or falls we shall get a combination of the phenomena always to be observed with three-component systems and the phenomena due to the changes at constant temperature. Which set of phenomena predominate will depend on the nature of the system studied and the rate of cooling.

So far we have only considered Case I $\mathrm{I} \alpha$, in which one of the assumptions was that the change of the $\beta$ into the a modification took place without heat effect. Inckily, the other two cases $I b$ and $I c$ can be dismissed with a very few words. If the change of the $\beta$ modification into the $\alpha$ modification be accompanied by evolution of heat, the curre for the equilibrium between the two modifications in the liquid phase before the addition of $\mathrm{C}$ nay be represented by $\mathrm{TX}$. If the addition of $\mathrm{C}$ displaces the equilibrimm very little if at all, the stable boundary curves will have the form of XR and $\mathrm{RC}$ in Fig. $\mathrm{I}$. This substitution of YX, XR and RC for YX, XS and SC is the only difference between the two cases. In the same way the diagram, Fig. I, will do duty for the case in which the $\beta$ modification changes into the $\alpha$ modification with absorption of heat prorided we replace YX, XS and SC by IXX, XT and TC.

In the case last considered, it will be noticed that the stable quadruple point ' $T$ ' lies very near the instable quintuple point $O$. This raises the question of the possibility of $\mathrm{X}^{\top} \mathrm{T}$ not cutting $\mathrm{HO}$ at all. If this were to happen, we should have a system classified under 2, with reversal of stability. The diagram for $2 a$ is giren in Fig. 2. The stable boundary curres are X., XS and $\mathrm{SC}$. Along $\mathrm{XN}$ the a modification is solid phase; along $\mathrm{NS}$ the $\beta$ modification and along SC the component $C$. The theorem of van Rijn van Alkemade enables ns to predict that the temperature will rise along the boundary curve from $\mathrm{S}$ to $\mathrm{N}$. 
The $\alpha$ modification is now the stable form only between $\mathrm{X}$ and $N$; while, between $X$ and $S$, the stable form is the $\beta$ modification. At $X$ we have a stable quadruple point with the two modifications as solid phases. It may be asked how this can come about in case, as is assumed here, there is no displacement of the equilibrium by the component $C$. So far as

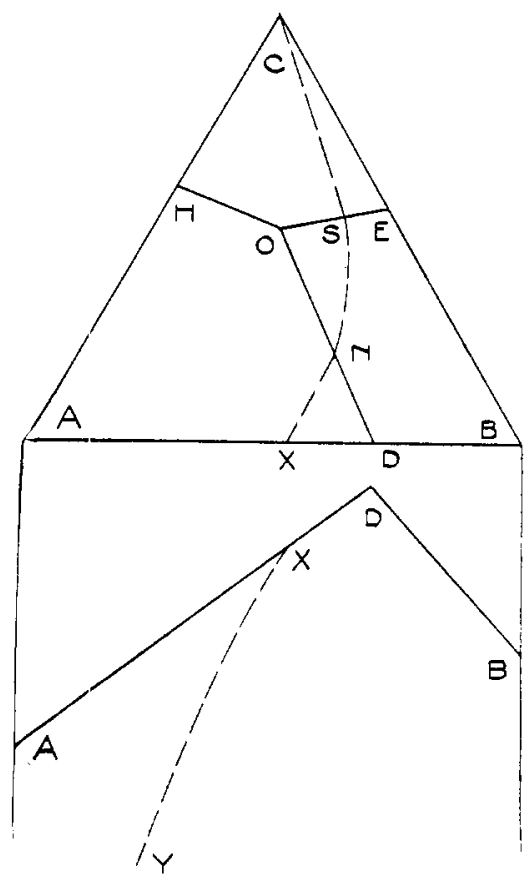

Fig. 2

can be seen at present, this cannot occur mincer these conditions m11less the two modifications can coexist as solid phases when the component $\mathrm{C}$ is not present. It should also be kept in ninind that the line XNSC need not be so much curved as in the diagran. It has been found by Carveth, ${ }^{2}$ while studying the system, potassium, lithinum and sodium nitrates, that a straiglit line can be drawn from the potassinn nitrate corner to the opposite side of the triangle, such that it cuts all three fields.

'Jour. Phys Chenr. 2, I92 (1S9s). 
The diagram for case $2 b$ wotnld not differ radically from Fig. 2 and therefore is not given. The chief point is that if the equilibrium between the two modifications is displaced by addition of component $\mathrm{C}$, the line VX may slant to the left instead of to the right. A distinction, which does not appear in the diagram, is that the displacement of the equilibrium may be sufficient to make the $\beta$ modification stable at temperatures at which it would change into the $a$ modification if the component C were not present.

The important point now is how far these theoretical predictions have been rerified experimentally. Cases corresponding to Fig. I nay be looked upon as normal and can probably be realized in the large majority of instances. Carveth ${ }^{\mathrm{I}}$ found that acetone does not displace the equilibrium between $\alpha$ and $\beta$ acetaldoxime towards the side of the $\beta$ modification; more than that his experiments did not show. It seems safe to assume that the diagram for benzaldoxime and water would correspond to one of the three types presented in Fig. I.

At least one case is known which connes under $\mathrm{I} b$, the dibenzorl acetone or acetyldibenzoyl methane, $\left(\mathrm{C}_{6} \mathrm{H}_{5} \mathrm{CO}\right)_{2} \mathrm{HC} \cdot \mathrm{COCH}$, studied by Claisen. ${ }^{2}$ From the second paper $^{3}$ it seems clear that the instable modification melts at about $85^{\circ}$, the stable modification at abont $I I 0^{\circ}$, while the temperature of the stable triple point is about $I O I-I O 2^{\circ}$. If the melt be heated above IIO ${ }^{\circ}$ and then cooled suddenly, the crystals are found to consist very largely of the instable form. This shows that, with rising temperature, the equilibrium is displaced toward the side of the less stable form. In other words, this latter changes into the more stable modification with evolution of heat. The addition of another component gives us at once a case coming under $I b$. While Claisen has not studied the subject from the point of view of the Phase Rule, he has made some interesting observations on the action of fifty percent aqueous alcohol. I quote his words, with

\footnotetext{
1 Jour. Phys. Chenn. 2, I66 (I895).

"Liebig's Annalen, 277, IS4 ( I893: : 29r, 25 (I896).

Not referred to in my first article. Jour. Phys. Chem, 2, I 43 (r8g8).
} 
the prefatory comment that he calls the instable form the a modification and the stable form the $\beta$ modification. ${ }^{1}$

"The $a$ compound changes quantitatively into the $\beta$ form if we dissolve it in boiling fifty percent alcohol and allow the solution to cool slowly. Consequently the $\beta$ modification can be recrystallized unchanged from hot fifty percent alcohol; if we cool the solution suddcnly by placing it in a freezing mixture or by pouring into ice water, the precipitate contains nearly ninety percent of the a componnd. I am not able to offer an explanation of this remarkable beharior." As a matter of fact, the explanation is an easy one to find on the basis of Duthem's theory. In the solution we have equilibriun between the $\alpha$ and the $\beta$ modification, the anount of the former being relatively large. If we cool slowly; the $a$ modification changes over as the $\beta$ crystals precipitate and the final result is pure $\beta$. If however we cool the solution suddenly, it becomes supersaturated with respect to both modifications and both precipitate. On longer standing the less stable form changes orer into the other. Claisen found also that a solution in hot absolute alcohol precipitated a mixture of the two forms on cooling. This mixture contained abont sixty-seren percent of the a modification. Althongh not so stated, I gather from the context that the rate of cooling in this case was gradual but not sogradual as in the first experiment with aqueous alcohol. This intermediate behavior is what would be expected uncler the circumstances. It seems probable that the rate of change is less in absolute than in aqueons alcohol though there are not data enough to establish this satisfactorily:

One case is known in which there is reversal of stability without the nature of the solvent being of much importance. Benzilorthocarboxylic acid, $\mathrm{C}_{6} \mathrm{H}_{5} \mathrm{CO} . \mathrm{CO} . \mathrm{C}_{6} \mathrm{H}_{4} \mathrm{COOH}$, occurs in two modifications, one yellow and the other white. ${ }^{2}$ The white or $\beta$ modification melss at $125^{-1} 30^{\circ}$ and changes slow $y$ into the rellow or $\alpha$ modification melting at I $4 \mathrm{I} .5^{\circ}$. The two modifica-

'I.iebig's Annalen, 29r, 30 (1896).

"Graebe. Ber. chem1. Ges, Berlin, 21, 2003 (1888); 23, I344 (1890). 
tions have the same molecular weight. By cristallizing at low temperatures, the yellow form can be converted into the white modification. Some experiments made in my laboratory by Mr. Soch have shown that the yellow crystals become white on standing, even when no solvent is present. Since the rellow form is stable at $100^{\circ}$ and the white at $20^{\circ}$, it is clear that there must be an inversion point somewhere between these two temperatures. This is now being investigated. ${ }^{x}$

As yet, I have not succeeded in finding any authenticated case in which the solvent forms no compound with the two modifications and yet reverses the stability. Displacement of equilibrinm occurs. The recent experiments of W. Wislicenus ${ }^{2}$ show this clearly. In no case, however, can one be certain that a reversal of stability has occurred. According to Beckmanu the high melting form of $p$-anisaldoxime does not change orer in boiling alcohol; ${ }^{3}$ but there is nothing to show that alcoliol makes the other form instable. In the same paper Beckmann states that $\beta$ benzaldoxime liquefies very rapidly in presence of traces of alcohol or water while benzene seems to have no effect. Here again, it is more than probable that we are dealing with a very low reaction velocity rather than with a change of stability. Similarly, Claisen found that dibenzoyl acetone could be recrystallized without difficulty from ligroin or benzene; but he would certainly have made more than a passing reference if he had observed a quantitative change of the less stable into the more stable form. It is much the same with all the other instances which I have yet come across. It minst, however, be kept in mind that the most farorable conditions for snch a trans-

${ }^{1}$ Some of Mr. Scch's measurements of the freezing-points show that Graebe's statements are not entirely accurate. From Graebe's papers one would certainly place benzilorthocarboxylic acid under $2 a$. As a matter of fact, the equilibrium phenomena are very different and much more complicater. The real analogy is with stufur. The statements in the text have been allowed to stand in order to emphasize the unreliability of the data compiled by people who were working without hypotheses to guide then.

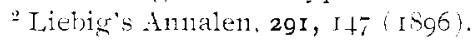

"Ber. chem. Ges. Berlin, 30, 1680 (1 990$)$.

"Liet)ig's Annalen, 29r, jo (IS96). 
formation are that the less stable form shall change into the more stable with absorption of heat and we have, as yet, no experimental knowledge of any sich case except, perhaps, in the two forms of benzilorthocarboxylic acid.

In this paper there lias been given a discussion of the conditions under which reversal of stability does and does not take place, on adding another component which forms no componnds with the two modifications. In this discussion it has been brought out that Duhem's theory is able to offer a simple explanation of what few facts are known and to predict many phenomena which have not yet been observed. In the third paper of the series I shall take up the question of teversal of stability when componinds are formed.

comell lniversity 